\title{
Snapback-Free Reverse-Conducting SOI LIGBT with an Integrated Self-Biased MOSFET
}

\section{Kemeng Yang}

University of Electronic Science and Technology of China

Jie Wei

University of Electronic Science and Technology of China

Kaiwei Dai

University of Electronic Science and Technology of China

Zhen Ma

University of Electronic Science and Technology of China

Congcong Li

University of Electronic Science and Technology of China

Xiaorong Luo ( $\nabla$ xrluo@uestc.edu.cn )

University of Electronic Science and Technology of China

\section{Research Article}

Keywords: lateral insulated gate bipolar transistor (LIGBT), snapback effect, reverse conducting, areaefficient

Posted Date: November 30th, 2021

DOI: https://doi.org/10.21203/rs.3.rs-1106070/v1

License: (c) (i) This work is licensed under a Creative Commons Attribution 4.0 International License.

Read Full License

Version of Record: A version of this preprint was published at Nanoscale Research Letters on April 18th, 2022. See the published version at https://doi.org/10.1186/s11671-022-03685-5. 


\title{
Snapback-Free Reverse-Conducting SOI LIGBT with an Integrated Self-Biased MOSFET
}

\author{
Kemeng Yang ${ }^{1 \#}$, Jie Wei ${ }^{1 \#}$, Kaiwei Dai ${ }^{1}$, Zhen $\mathrm{Ma}^{1}$, Congcong $\mathrm{Li}^{1} \&$ Xiaorong Luo ${ }^{1 *}$ \\ ${ }^{1}$ the State Key Laboratory of Electronic Thin Films and Integrated Devices, \\ University of Electronic Science and Technology of China, Chengdu 610054, China
}

\begin{abstract}
A novel snapback-free RC-LIGBT with integrated self-biased N-MOSFET is proposed and investigated by simulation. The device features an integrated self-biased N-MOSFET(ISM) on the anode active region. One side of the ISM is shorted to the P+ anode electrode of RC-LIGBT and the other side is connected to the $\mathrm{N}+$ anode via a floating ohmic contact. The adaptively turn-on/off of the ISM contributes to improve the static and dynamic performance of the ISM RC-LIGBT. In the forward-state, due to the off-state of the ISM, the snapback could be effectively suppressed without requiring extra device area compared with the SSA (separated shorted anode) and STA (segmented trenches in the anode) LIGBTs. In the reverse conduction, the ISM is turned on and the parasitic NPN in the ISM is punched through, which provides a current path for the reverse current. Meanwhile, during the turn-off and reverse recovery states, the ISM turns on, providing a rapid electron extraction path. Thus, a superior tradeoff between the on-state voltage drop $\left(V_{\text {on }}\right)$ and turnoff loss $\left(E_{\text {off }}\right)$ as well as an improved reverse recovery characteristic can be obtained. Compared with the STA device, the proposed ISM RC-LIGBT reduces $E_{\text {off }}$ by $21.5 \%$ without snapback. Its reverse recovery charge is reduced by $53.7 \% / 58.6 \%$ compared to that of the SSA LIGBT with $L_{\mathrm{b}}=40 / 60 \mu \mathrm{m}$ at the same $V_{\text {on. }}$. Due to the prominent static and dynamic characteristic, the power loss of ISM RC-LIGBT in a completed switching cycle is reduced.
\end{abstract}

Keywords lateral insulated gate bipolar transistor (LIGBT), snapback effect, reverse conducting, area-efficient

\section{Introduction}

The lateral insulated gate bipolar transistor (LIGBT) on silicon-on-insulator (SOI) technology is an attractive device to be used in three-phase single chip inverter ICs due to its low on-state voltage $\left(V_{\text {on }}\right)$ under high current density and easy integration ${ }^{[1]-[3]}$. However, the unidirectional switch characteristic requires the conventional LIGBT connecting in anti-parallel with a diode to conduct the reverse current, which introduces stray inductance and the extra chip area ${ }^{[4]-[5]}$. The usage of the shorted anode (SA) LIGBT, instead of the conventional LIGBT with an antiparallel diode in the switching modules, proves to be an effective method to address drawbacks as motioned above ${ }^{[6]-[8]}$. Meanwhile, the introduced $\mathrm{N}+$ anode in the SA LIGBT also provides an electron extraction path and avoids the long current tail during turn-off, resulting in a small turn-off loss. However, the introduced $\mathrm{N}+$ anode also makes the SA LIGBT suffers from the undesirable snapback effect, which may lead to the device reliability problems.

To suppress the snapback effect, many structures are proposed. The separated SA LIGBT (SSA LIGBT) alleviates the snapback by increasing the space and the distributed resistance between the $\mathrm{P}+$ anode and $\mathrm{N}+$ anode ${ }^{[9]}$, while it needs a large device area to effectively eliminate the snapback effect. The segmented trenches in the anode (STA) LIGBT ${ }^{[10]}$ and trench barrier shorted anode (TBSA) LIGBT ${ }^{[1]}$ decrease the distance between $\mathrm{N}+$ and $\mathrm{P}+$ anode by implementing deep oxide trenches at

Xiaorong Luo* is the corresponding author(e-mail: xrluo@uestc.edu.cn). Kemeng Yang ${ }^{\#}$ and Jie Wei" contribute equally. 
anode side, while the process is difficult and also increases the cost. Multi-gates devices show better performance by controlling the anode gate, but it needs complex controlling circuits ${ }^{[12]-[15]}$. Integrating a diode in the LIGBT to realize reverse conduction and suppress snapback is a smart method, yet the schottky barrier diode makes the performance of the device to be influenced by the temperature ${ }^{[16]}$.

In this paper, a novel area-efficient and snapback-free RC-LIGBT with Integrated Self-biased MOSFET (ISM) is proposed and its mechanism is investigated by Sentaurus TCAD tools. The adaptively turned-on/off ISM leads to the snapback-free forward conduction characteristic, a decreased turn-off loss and a superior reverse recovery characteristic. As a result, the proposed device performs a reduced power loss in a completed switching cycle.

\section{Device Structure and Mechanism}

Fig. 1(a) shows the schematic of the proposed RC LIGBT featured an Integrated Self-biased MOSFET. The integrated MOSFET on the top of the anode active region does not need extra device area. One side of the ISM is shorted to the $\mathrm{P}+$ anode and the other side is connected to the $\mathrm{N}+$ anode via a floating ohmic contact (FOC), as illustrated in Fig. 1(b). It is worth noting that the ISM is self-adaptively turned on/off according to the operation status of the RC-LIGBT. As Fig. 1(b) denoted, $N_{\mathrm{P}}$ and $L_{\mathrm{P}}$ are the doping concentration and length of the $\mathrm{P}$ region in ISM, respectively. $t_{\mathrm{OX}}$ is the thickness of the oxide of ISM. $N_{\mathrm{A}}$ and $N_{\mathrm{N}}$ are the doping concentration of $\mathrm{P}+$ anode and N-buffer, respectively.

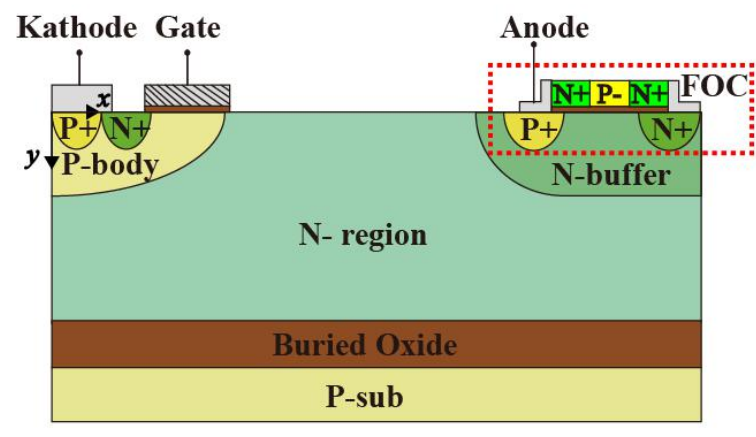

(a)

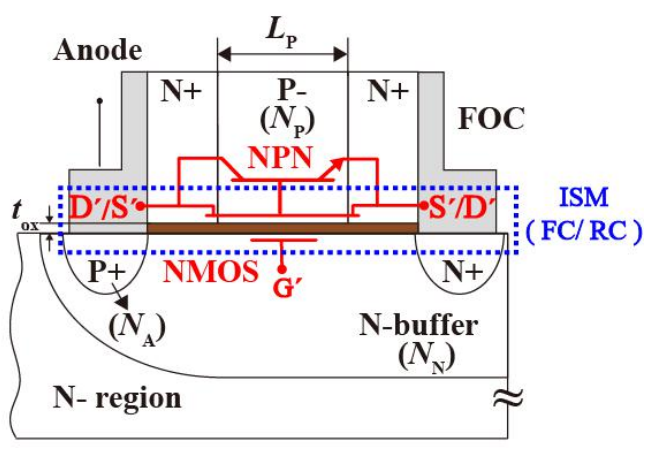

(b)

Fig. 1 (a) Schematic cross section of the ISM RC-LIGBT; (b) zoomed-in schematic cross section of the anode side.

Fig. 2 gives the operation mechanism of the ISM RC-LIGBT. Although the ISM is regarded as a self-biased MOSFET, the influence of parasitic NPN in the ISM cannot be ignored. Therefore, in the following analysis, the states of self-biased MOS and the open base NPN are both discussed. The equivalent circuits of ISM RC-LIGBT at Forward/ Reverse Conducting (FC/RC) states are shown in Fig. 2(a) and (b), respectively. It reveals that the operation state of the ISM influences the current path. Fig. 2(c) summarizes the states of the ISM and the open base NPN under different operation states of the ISM RC-LIGBT. Followings are the detailed explanation: 


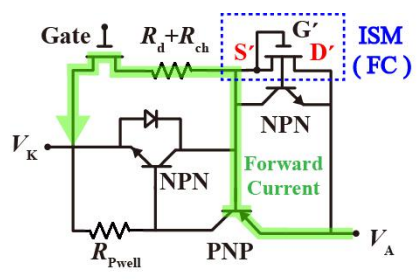

(a)

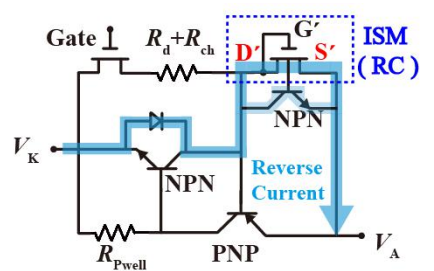

(b)

\begin{tabular}{cccc}
\hline $\begin{array}{c}\text { LIGBT } \\
\text { State }\end{array}$ & $\begin{array}{c}\text { Self-biased } \\
\text { MOS }\end{array}$ & $\begin{array}{c}\text { Open base } \\
\text { NPN }\end{array}$ & $\begin{array}{c}\text { LIGBT } \\
\text { Performance }\end{array}$ \\
\hline $\begin{array}{c}\text { Forward Conduction } \\
\left(0<V_{\mathrm{AK}} \leq V_{\mathrm{PN}}\right)\end{array}$ & $\begin{array}{c}\text { off } \\
V_{\mathrm{G} \text { ' }} \approx 0<V_{\mathrm{T}}\end{array}$ & off & $\begin{array}{c}\text { Suppress } \\
\text { Snapback }\end{array}$ \\
$\begin{array}{c}\text { Reverse Conduction } \\
\left(V_{\mathrm{AK}}<0\right)\end{array}$ & $\begin{array}{c}\text { on } \\
V_{\mathrm{G} \mathrm{S}^{\prime}}>V_{\mathrm{T}}\end{array}$ & on & Realize RC \\
$\begin{array}{c}\text { Turn-off } \\
\left(V_{\mathrm{AK}}>0\right)\end{array}$ & $\begin{array}{c}\text { off } \\
V_{\mathrm{G}^{\prime} \mathrm{S}} \approx 0<V_{\mathrm{T}}\end{array}$ & on & Decrease $E_{\mathrm{off}}$ \\
Reverse Recovery & off & & \\
$\left(V_{\mathrm{AK}}>0\right)$ & $V_{\mathrm{G}^{\prime} \mathrm{S}} \approx 0<V_{\mathrm{T}}$ & on & Decrease $Q_{\mathrm{Tr}}$ \\
\hline
\end{tabular}

(c)

Fig. 2 Equivalent circuit of the ISM RC-IGBT for (a) FC state and (b) RC state. (c) states of the ISM under different operation states of LIGBT. Here $V_{\mathrm{PN}}$ is the built-in potential of $\mathrm{P}+/ \mathrm{N}-$ buffer junction, $V_{\mathrm{T}}$ is the threshold voltage of the self-biased MOS.

In the forward conduction, the ISM and the parasitic NPN are in off-state when the P+/ N-buffer junction turns on. In this case, the current flowlines only derive from the $\mathrm{P}+$ anode and the device operates in the bipolar mode, as shown in the Fig. 3(a). When the $N_{\mathrm{p}}$ vale is too small, the P region in the ISM is fully depleted and then electrons are swept through the NPN, as shown in Fig. 3(b). The current flowlines go through the NPN region, while the P+/N-buffer junction is still in off-state. In this case, the device is in unipolar mode at the initial forward-conducting state, resulting in snapback, which should be avoided.

In the reverse conduction, the ISM and parasitic NPN are adaptively turned on, providing a reverse conducting path and realize RC. Fig. 4 shows the reverse-conducting current flowlines. With the increasing $\left|V_{\mathrm{AK}}\right|$, the ISM undergoes three cases: the expansion of depleted region near the channel and the ISM is in off-state, as shown in Fig. 4(a); inversion layer in the ISM is formed and the ISM turns on, as shown in Fig. 4(b); and both the ISM and the parasitic NPN are in on-state, as shown in Fig. 4(c). The turned-on ISM is vital to realize the reverse-conducting for the RC-LIGBT.

During turn-off and reverse recovery period, the large voltage between anode and cathode triggers the parasitic NPN in the ISM and then electrons are swept through the NPN, providing an additional electron extracting path. As a result, the extra electrons stored in the drift region could be rapidly removed and the ISM LIGBT achieves a low turn-off loss and reverse recovery charges.

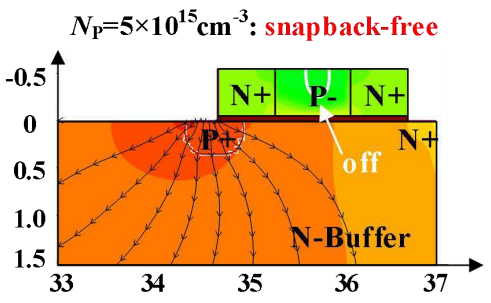

(a)

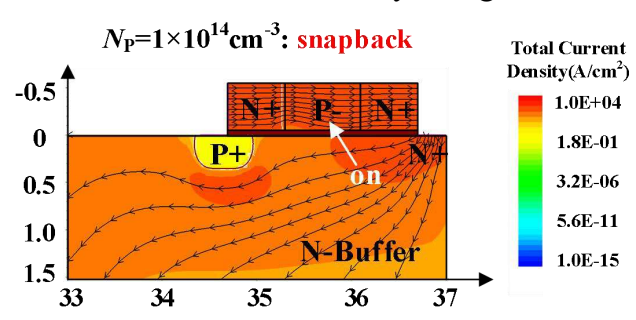

(b)

Fig. 3 Current flowlines in the forward-conducting state for the ISM RC-LIGBT $\left(L_{\mathrm{P}}=0.8 \mu \mathrm{m}, t_{\mathrm{ox}}=50 \mathrm{~nm}, @ V_{\mathrm{AK}}=1 \mathrm{~V}\right)$ : (a) $N_{\mathrm{P}}=5 \times 10^{15} \mathrm{~cm}^{-3}$; (b) $N_{\mathrm{P}}=1 \times 10^{14} \mathrm{~cm}^{-3}$. 


$$
\text { a) } V_{\mathrm{AK}}=-0.5 \mathrm{~V}
$$

self-biased MOS: off

open-based NPN: off

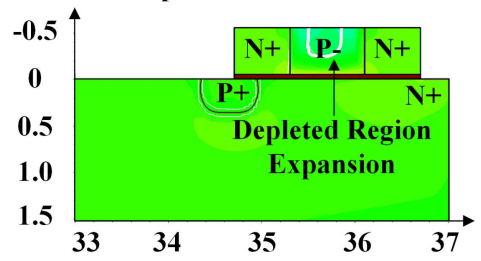

(a)

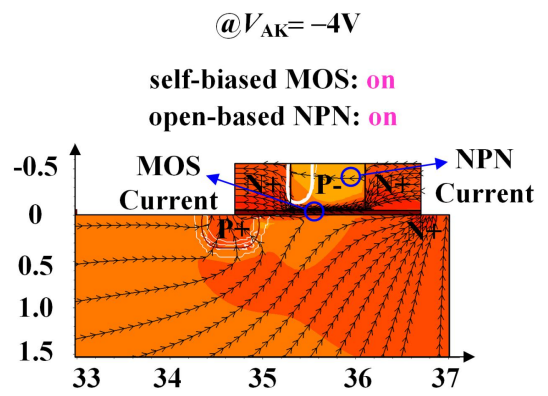

(c)

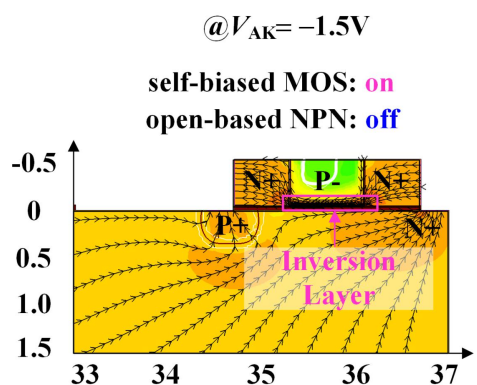

(b)

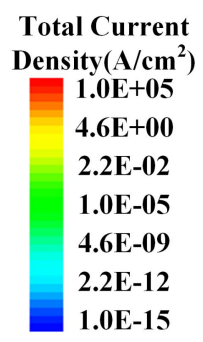

Fig. 4 Current flowlines in the reverse-conducting state for the ISM RC-LIGBT $\left(N_{\mathrm{P}}=1 \times 10^{16} \mathrm{~cm}^{-3}, L_{\mathrm{P}}=0.8 \mu \mathrm{m}, t_{\mathrm{OX}}=50 \mathrm{~nm}\right):(\mathrm{a})$ $V_{\mathrm{AK}}=-0.5 \mathrm{~V}$; (b) $V_{\mathrm{AK}}=-1.5 \mathrm{~V} ;$ (c) $V_{\mathrm{AK}}=-4 \mathrm{~V}$.

\section{Results and Discussion}

In order to verify the operation mechanisms and advantages of the proposed ISM RC LIGBT, SSA/STA LIGBTs and the conventional LIGBTs with an anti-parallel diode are also simulated and compared. The optimized parameters for each device in simulation are listed in Table 1. All devices are designed with a forward blocking capability of $300 \mathrm{~V}$.

Table 1 Key Parameters for LIGBTs

\begin{tabular}{ccccc}
\hline Parameters & ISM & CON & SSA & STA \\
\hline SOI layer thickness, $t_{\mathrm{s}}(\mu \mathrm{m})$ & 6 & 6 & 6 & 6 \\
Buried oxide thickness, $t_{\mathrm{BOX}}(\mu \mathrm{m})$ & 3 & 3 & 3 & 3 \\
N-drift doping, $N_{\mathrm{d}}\left(\times 10^{15} \mathrm{~cm}^{-3}\right)$ & 3.6 & 3.6 & 3.6 & 3.6 \\
N-drift length, $L_{\mathrm{d}}(\mu \mathrm{m})$ & 30 & 30 & 30 & 30 \\
N-buffer doping, $N_{\text {buffer }}\left(\times 10^{17} \mathrm{~cm}^{-3}\right)$ & 2 & 2 & 2 & 2 \\
Doping of the N+ region in the ISM $\left(\times 10^{18} \mathrm{~cm}^{-3}\right)$ & 1 & --- & --- & --- \\
Length of the N+ region in the ISM $(\mu \mathrm{m})$ & 0.6 & --- & --- & -- \\
Spacing between trenches, $S_{\mathrm{T}}(\mu \mathrm{m})$ & --- & --- & --- & 0.5 \\
Distance between P+ and $\mathrm{N}+$ anode, $L_{\mathrm{b}}(\mu \mathrm{m})$ & 1 & --- & $40 / 60$ & 5 \\
\hline
\end{tabular}

Fig. 5 shows the conduction characteristics of different type LIGBTs. In the forward conduction, the ISM RC-LIGBT with optimal $N_{\mathrm{P}}$ and $L_{\mathrm{P}}$ can eliminate snapback without increasing the $V_{\text {on }}$. For the SSA LIGBT, even though the $L_{\mathrm{b}}$ achieves $40 \mu \mathrm{m}$, the snapback effect still exists and its $V_{\text {on }}$ is much larger than that of the ISM RC-LIGBT. The STA LIGBT could suppress the snapback, but it still degenerates the $J_{\mathrm{A}}$ and the $V_{\mathrm{on}}$ is larger than ISM RC-LIGBT. The proposed structure features a better forward conduction characteristic than the other LIGBTs with a shorted anode. At the 
reverse-conducting state, the $V_{\mathrm{F}}=1.8 \mathrm{~V}$ for the ISM LIGBT at $J_{\mathrm{A}}=100 \mathrm{~A} / \mathrm{cm}^{2}$ is higher than $1.62 \mathrm{~V}$ of SSA LIGBT with $L_{\mathrm{b}}=60 \mu \mathrm{m}$, because the RC current path from the cathode to anode in the ISM RC-LIGBT flows through a diode and an NMOS in series.

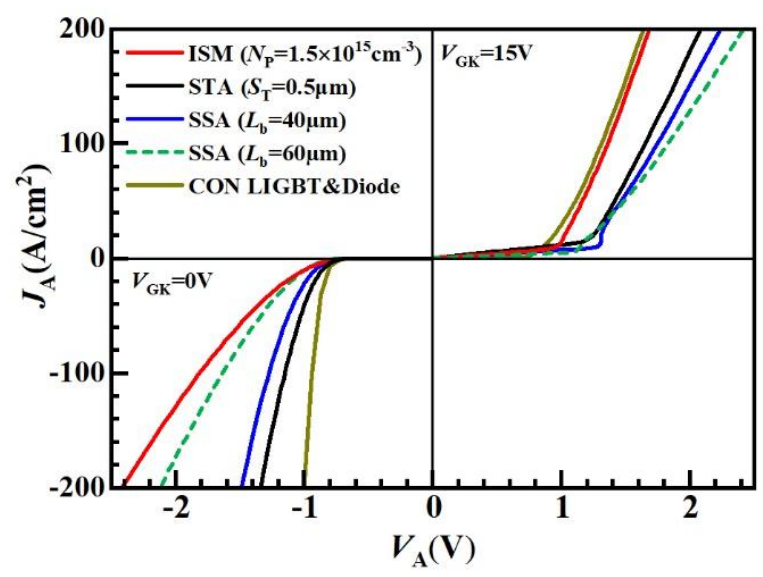

Fig. 5 Comparison of conduction characteristics for ISM, STA, SSA LIGBTs and Con. LIGBT\& diode. $S_{\mathrm{T}}$ is the spacing between the segmented trenches for the STA LIGBT. $L_{\mathrm{b}}$ is the distance between N-buffer and separated N + anode for the SSA LIGBT. For ISM device, $N_{\mathrm{P}}=1.5 \times 10^{15} \mathrm{~cm}^{-3}, L_{\mathrm{P}}=0.8 \mu \mathrm{m}$.

Fig. 6(a) depicts the influence of key parameters of $N_{\mathrm{P}}, L_{\mathrm{P}}$ and $t_{\mathrm{OX}}$ on conduction characteristics for ISM RC-IGBT. With the increasing $N_{\mathrm{P}}$ or $L_{\mathrm{P}}$, the snapback effect is weakened and even eliminated in the red and blue lines. Whereas, the devices with $t_{\mathrm{OX}}=50$ and $100 \mathrm{~nm}$ exhibit the same forward conduction characteristics owing to the hardly turned-on ISM at FC state. Thus, the forward-conducting characteristic of ISM RC-LIGBT mainly depends on the state of the parasitic NPN. To suppress the snapback, the $\mathrm{P}+/ \mathrm{N}-$ buffer junction should be turned on earlier than the parasitic NPN, namely

$$
V_{\mathrm{PN}} \leq V_{\mathrm{PT}}
$$

where $V_{\mathrm{PT}}$ is the punch-through voltage of parasitic NPN in the ISM. Taking formulas of $V_{\mathrm{PN}}$ and $V_{\mathrm{PT}}{ }^{[17]}$ into Eq., the parameters of the ISM should satisfy

$$
N_{\mathrm{P}} L_{\mathrm{P}}^{2} \geq \frac{2 \varepsilon_{\mathrm{S}} k T}{q^{2}} \ln \left(\frac{N_{\mathrm{A}} N_{\mathrm{N}}}{n_{i}^{2}}\right)
$$

where $n_{\mathrm{i}}$ is the intrinsic carrier concentration, $q$ is the electronic charge, $\varepsilon \mathrm{s}$ is dielectric constant of $\mathrm{Si}, k$ is the Boltzmann constant, and $T$ is the absolute temperature. In the RC state, with the decreasing $N_{\mathrm{P}}$, $L_{\mathrm{P}}$ or $t_{\mathrm{OX}}$, the diode-mode forward voltage $\left(V_{\mathrm{F}}\right)$ of ISM RC-LIGBT is reduced. Both the punched-through NPN and the turned-on ISM contribute to the increase in the reverse current.

Fig. 6(b) illustrates the influence of the $N_{\mathrm{P}}$ and $L_{\mathrm{P}}$ on the $\Delta V_{\mathrm{SB}}$ and denotes the snapback-free region. It is worth noting that the optimal value of $N_{\mathrm{P}}$ for $\mathrm{FC}$ and $\mathrm{RC}$ state is the opposite trend. Thus, the optimized relationship of $N_{\mathrm{P}}$ and $L_{\mathrm{P}}$ could be obtained when the equal sign is taken in Eq., namely the boundary of snapback-free region in Fig. 6(b). Fig. 6(b) also reveals that the optimal relation of $N_{\mathrm{P}}$ and $L_{\mathrm{P}}$ obtained by simulation is consistent with the calculated results of the model. Therefore, the model could provide a guidance to select the appropriate values of the $N_{\mathrm{P}}, L_{\mathrm{P}}, N_{\mathrm{A}}$ and $N_{\mathrm{N}}$ so as to achieve a snapback-free device. 


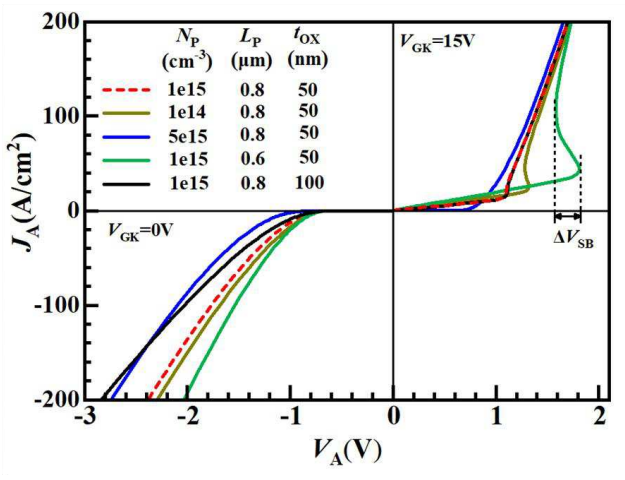

(a)

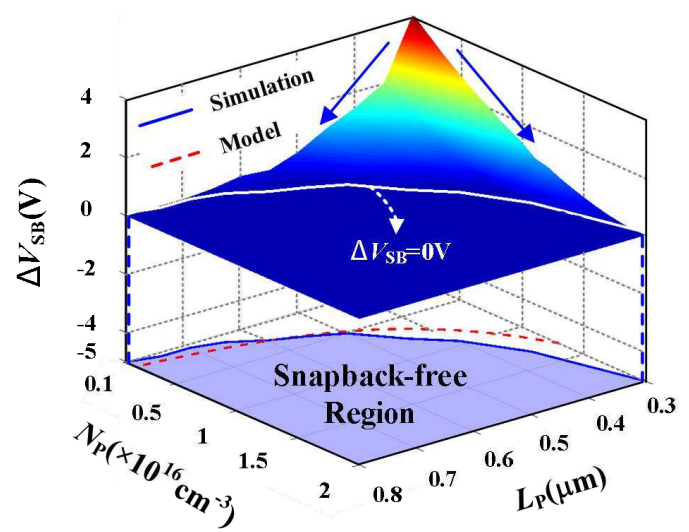

(b)

Fig. 6 (a) Influence of the $N_{\mathrm{P}}, L_{\mathrm{P}}$ and $t_{\mathrm{OX}}$ on conduction characteristics. (b) 3-D graph of influence of the $N_{\mathrm{P}}$ and $L_{\mathrm{P}}$ on the $\Delta V_{\mathrm{SB}}$.

Fig. 7(a) gives the turn-off waveforms of different LIGBTs. The ISM RC-LIGBT turns off the fastest and SSA LIGBT has the longest turn-off time due to the extra $L_{\mathrm{S}}$ region. To investigate the turn-off mechanism of ISM RC-LIGBT, Fig. 7(b) gives the electron current flowlines at point A in Fig. 7(a). It reveals that the parasitic NPN in the ISM is adaptively turned on. It provides an additional path to rapidly remove the electrons from the drift region. This is constructive to reduce the $E_{\text {off. }}$ In terms of the SSA and STA LIGBT, the long or narrow electron extraction path weakens the effect of reducing $E_{\text {off. }}$ Thus, the ISM RC-IGBT features a better $E_{\text {off }}-V_{\text {on }}$ tradeoff performance than SSA and STA LIGBTs, as shown in Fig. 8. The $E_{\text {off }}$ of ISM RC-LIGBT is reduced by $21.5 \%$ compared to that of the STA LIGBT with the same $V_{\text {on }}$ at $J_{\mathrm{A}}=100 \mathrm{~A} / \mathrm{cm}^{2}$. Compared with the SSA LIGBT with $L_{\mathrm{b}}=40 / 60 \mu \mathrm{m}$, ISM LIGBT reduces $V_{\text {on }}$ by $13.3 \% / 20.8 \%$ at the same $E_{\text {off, because ISM RC-LIGBT does not occupy }}$ additional area to eliminate the snapback. Even at higher turn-off current density of $200 \mathrm{~A} / \mathrm{cm}^{2}$, the ISM LIGBT still exhibits better tradeoff performance than SSA and STA LIGBTs, as illustrated in Fig. 8 (b).

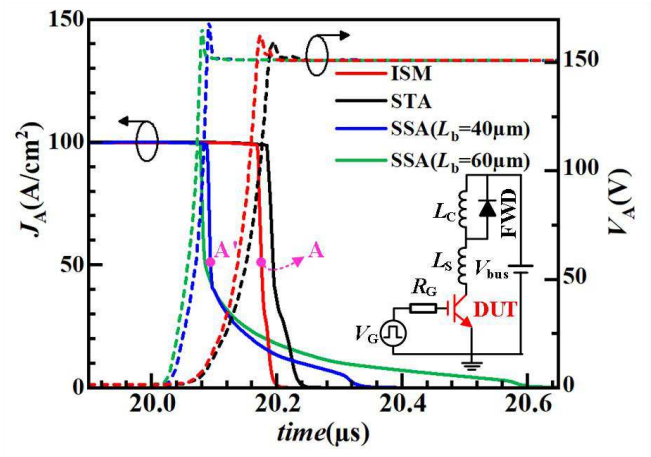

(a)

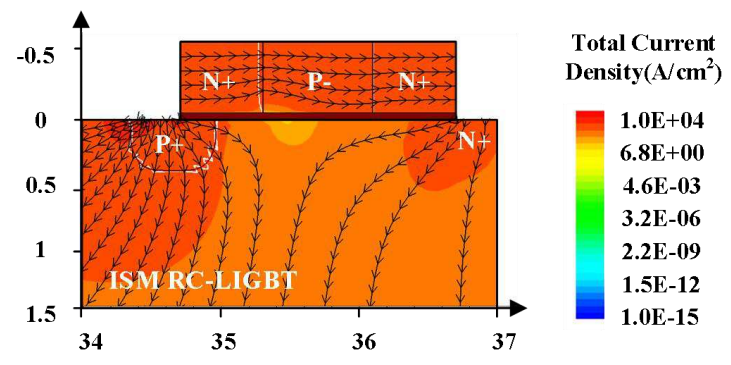

(b)

Fig. 7 (a) Turn-off waveforms under clamped inductive load with $L_{\mathrm{C}}=4 \mu \mathrm{H}, L_{\mathrm{S}}=1 \mathrm{nH}, R_{\mathrm{G}}=10 \Omega, V_{\text {bus }}=150 \mathrm{~V}, V_{\mathrm{on}}=1.5 \mathrm{~V}$. (b) Electron Current flowlines for the ISM RC-LIGBT at anode side [at point A in (a)]. 


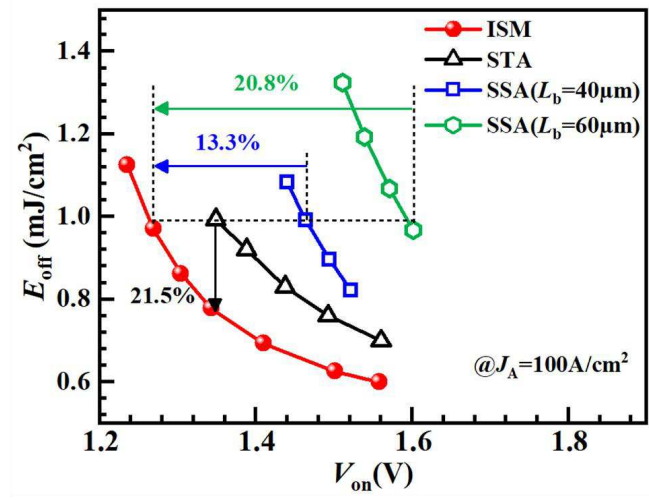

(a)

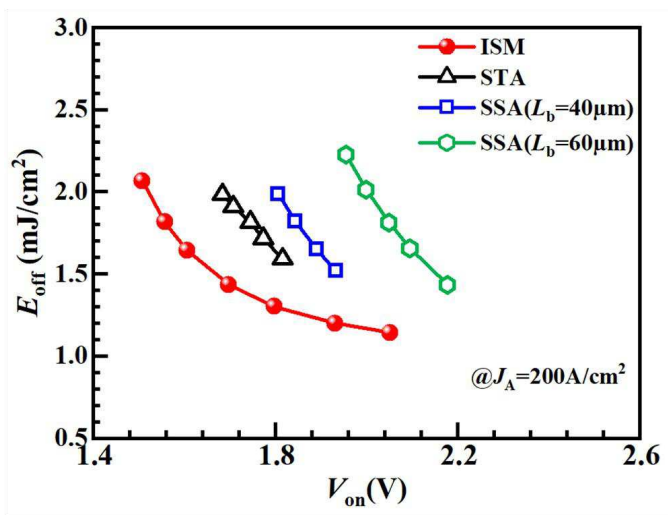

(b)

Fig. $8 E_{\text {off }}$ versus $V_{\text {on }}$ for different LIGBTs: (a) $J_{\mathrm{A}}=100 \mathrm{~A} / \mathrm{cm}^{2}$ and (b) $J_{\mathrm{A}}=200 \mathrm{~A} / \mathrm{cm}^{2}$.

Fig. 9 compares the reverse recovery characteristics of the ISM RC-LIGBT, SSA LIGBT and the conventional LIGBT with an anti-parallel diode. The test circuit is shown in Fig. 9 (a). In Fig. 9 (b), the reverse recovery charge $Q_{\text {rr }}$ of the ISM RC-LIGBT is $13.3 \%$ lower than that of the conventional LIGBT with an antiparallel diode, due to its $30 \%$ reduced reverse recovery peak current. Compared with the SSA LIGBT $\left(L_{\mathrm{b}}=40 / 60 \mu \mathrm{m}\right)$ device, $Q_{\text {rr }}$ of ISM RC-LIGBT is reduced by $53.7 \% / 58.6 \%$. Fig. $9(\mathrm{c})$ gives the electron distribution in the $x$-dimension from $t_{1}$ to $t_{4}$ period (denoted in Fig. 9(b)). Compared with the SSA LIGBT, the ISM RC-LIGBT extracts the electrons more quickly, since the punched-through parasitic NPN in the ISM contributes to a fast electron extraction from drift region. In addition, the ISM RC-LIGBT does not introduce extra electron storage region $L_{\mathrm{b}}$ as the SSA LIGBT does. Therefore, ISM RC-LIGBT exhibits a better reverse recovery performance than the SSA LIGBT.

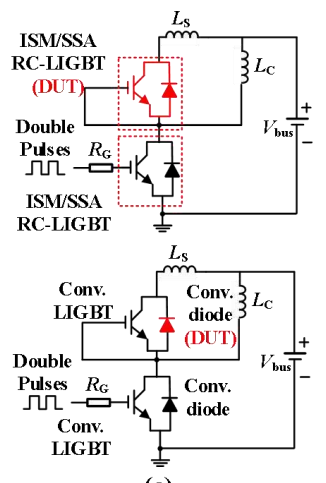

(a)

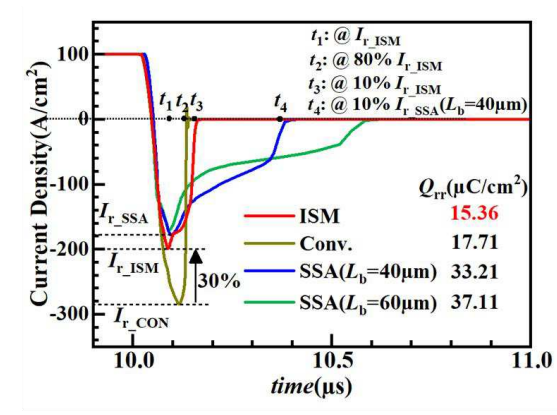

(b)

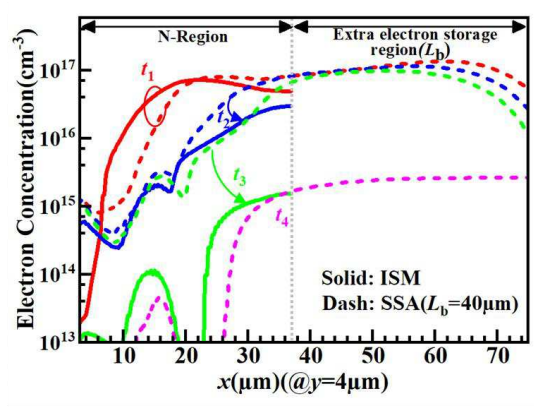

(c)

Fig. 9 Reverse recovery characteristics of the ISM RC-LIGBT, SSA LIGBT and the conventional LIGBT with an anti-parallel diode: (a) circuit diagram for reverse recovery $\left(L_{\mathrm{C}}=1 \mathrm{mH}, L_{\mathrm{S}}=1 \mathrm{nH}, R_{\mathrm{G}}=25 \Omega, V_{\text {bus }}=200 \mathrm{~V}, V_{\mathrm{on}}=2 \mathrm{~V}\right)$; (b) reverse recovery waveforms; (c) electron concentration distribution along $y=4 \mu \mathrm{m}$.

In order to investigate the characteristic of ISM RC-LIGBT, the waveforms in a complete on/off switching cycle are compared for the ISM RC-LIGBT, STA and SSA LIGBT $\left(L_{\mathrm{b}}=60 \mu \mathrm{m}\right)$ in Fig. 10. The circuit diagram is shown in Fig. 9(a) and the device under test is RC-IGBT2. During the turn-on period, the current overshoot is caused by the reverse recovery of RC-LIGBT1. Compared with the STA LIGBT, ISM RC-LIGBT has a smaller current peak. It also owns a shorter current tail than that of the SSA LIGBT. This is owing to the better reverse recovery characteristic of ISM RC-LIGBT as illustrated in Fig. 9. During the turn-off period, the ISM RC-LIGBT has the shortest turn-off time, as shown in Fig. 10. 


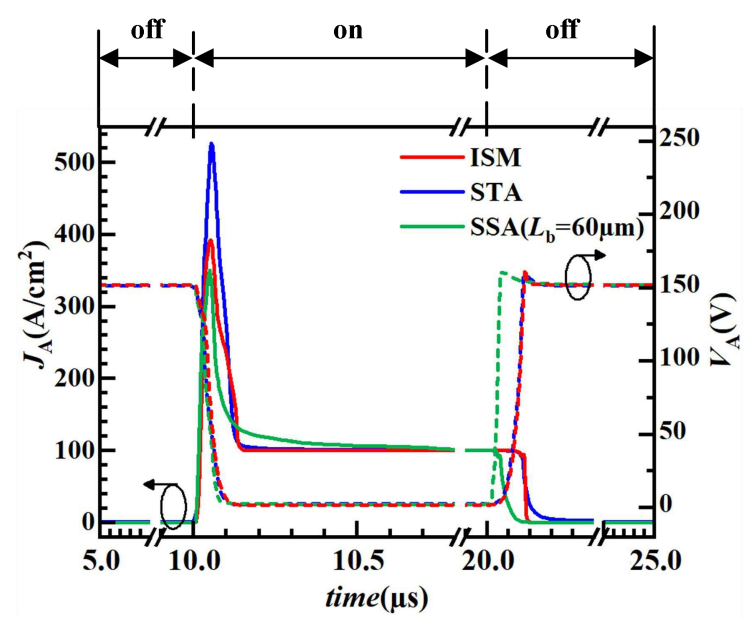

Fig. 10 The waveforms of the ISM RC-LIGBT, STA and SSA LIGBT in a complete switching cycle. $\left(L_{\mathrm{C}}=1 \mathrm{mH}, L_{\mathrm{S}}=1 \mathrm{nH}\right.$, $\left.R_{\mathrm{G}}=10 \Omega, V_{\text {bus }}=150 \mathrm{~V}, N_{\mathrm{A}}=6 \times 10^{17} \mathrm{~cm}^{-3}\right)$

Fig. 11 obtains the power loss for the three devices at different frequencies. The total power loss of the RC-LIGBT2 includes the power loss of turn-on/off $\left(P_{\mathrm{on}} / P_{\text {off }}\right)$ and the conduction power loss $\left(P_{\text {con }}\right)$. The power loss calculations of the three parts can be given by

$$
\begin{gathered}
P_{\text {on } / \text { off }}=\frac{1}{t_{\text {total }}} \sum_{n=1}^{N} E_{\text {on } / \text { off }}=\frac{1}{t_{\text {total }}} \sum_{n=1}^{N} \int_{t^{\prime}}^{t^{\prime}+t_{\text {on off }}} V_{\mathrm{AK}} J_{\mathrm{A}} d t \\
P_{\text {con }}=\frac{1}{t_{\text {total }}} \sum_{n=1}^{N} E_{\text {con }}=\frac{1}{t_{\text {total }}} \sum_{n=1}^{N} V_{\text {on }} J_{\mathrm{L}} t_{\text {con }}
\end{gathered}
$$

where the summation index $N$ corresponds to the times of switching actions in total current commutation time $t_{\text {total }}$. Here $t_{\text {on }}, t_{\text {off }}$ and $t_{\text {con }}$ represent the time of turn-on/off and the forward conduction. $t^{\prime}$ is the start time of the three states and $J_{\mathrm{L}}$ is the loading current density. According to Fig. 11, the ISM RC-LIGBT has the lowest power loss among three types of LIGBTs. Since the $V_{\text {on }}$ of ISM RC-LIGBT is the smallest among these three devices, as illustrated in Fig. 6, the ISM RC-LIGBT has the lowest $P_{\text {con. }}$. While, with the enhanced injection, the turn-on/off loss of the ISM device does not increase a lot. Therefore, when the operation frequency $\left(f_{\mathrm{s}}\right)$ increases to $50 \mathrm{kHz}$, the power loss of ISM RC-LIGBT still decreases by $29 \%$ compared with that of STA LIGBT. Fig. 12 compares the power losses with various LIGBTs at the rated $J_{\mathrm{L}}$ ranging from 100 to $300 \mathrm{~A} / \mathrm{cm}^{2}$. Although a high density of current leads to an increased power loss, the ISM RC-LIGBT still performs a lowest power loss at $J_{\mathrm{L}}$ $=300 \mathrm{~A} / \mathrm{cm}^{2}$ among three LIGBTs.

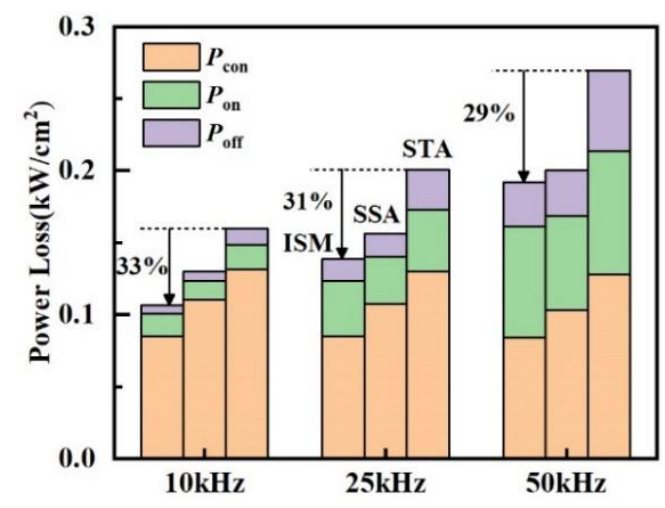

Fig. 11 Power losses under different switching frequencies of the ISM RC-LIGBT, STA and SSA LIGBT at $J_{\mathrm{L}}=100 \mathrm{~A} / \mathrm{cm}^{2}$ within 1 second. 


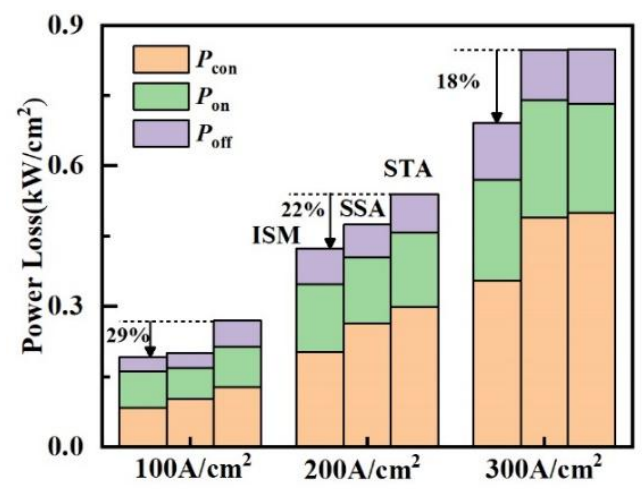

Fig. 12 Power losses under different current densities of the ISM RC-LIGBT, STA and SSA LIGBT at $f_{\mathrm{s}}=50 \mathrm{kHz}$ within 1 sencond.

\section{Conclusion}

The ISM RC-LIGBT with an integrated self-biased MOS on the top of the anode active region is proposed and investigated. The novel ISM does not occupy extra device area. By optimizing the parameters of the ISM, the ISM RC-LIGBT could eliminate the snapback effect without degrading the forward conduction characteristic. Meanwhile, the adaptively turned-on ISM provides a current path for reverse-conducting current. The parasitic NPN in the ISM also gives an additional electron extra path during the turn-off and reverse recovery. Thus, the proposed device achieves a better tradeoff relationship between $V_{\text {on }}$ and $E_{\text {off, }}$ as well as better reverse recovery characteristic. Compared with SSA LIGBT $\left(L_{\mathrm{b}}=40 / 60 \mu \mathrm{m}\right)$, it decreases $V_{\text {on }}$ by $13.3 \% / 20.8 \%$ at the same $E_{\text {off. Moreover, it also features a }}$ lower $Q_{\mathrm{rr}}$ and a better reverse recovery characteristic. Compared with STA LIGBT, the power loss of ISM RC-LIGBT in a completed on/off switching cycle is decreases by $29 \%$ at $J_{\mathrm{L}}=100 \mathrm{~A} / \mathrm{cm}^{2}, f_{\mathrm{s}}=50 \mathrm{kHz}$. This reveals that the proposed device could reduce the power loss in the application.

\section{Abbreviations}

LIGBT: lateral insulated gate bipolar transistor; SOI: silicon-on-insulator; SA LIGBT: shorted anode LIGBT; SSA LIGBT: separated SA LIGBT; STA LIGBT: segmented trenches in the anode LIGBT; TBSA LIGBT: trench barrier shorted anode LIGBT; ISM: Integrated Self-biased MOSFET

\section{Acknowledgements}

This work was supported by the National Natural Science Foundation of China 62104030, the China Postdoctoral Science Foundation 2021M690543, the Guangdong Basic and Applied Basic Research Foundation 2019A1515110950, National Natural Science Foundation of China 62004031

\section{Funding}

National Natural Science Foundation of China 62104030, the China Postdoctoral Science Foundation 2021M690543, the Guangdong Basic and Applied Basic Research Foundation 2019A1515110950, National Natural Science Foundation of China 62004031

\section{Authors' contributions}

Kemeng Yang, Jie Wei and Xiaorong Luo designed the concept and analysis. Daikai Wei, Zhen Ma and Congcong Li conducted the simulation. Kemeng Yang, Daikai Wei and Zhen Ma conducted data processing and wrote the main manuscript. Kemeng Yang, Jie Wei and Xiaorong Luo modified the paper. All authors read and approved the final manuscript.

\section{Availability of Data and Materials}

The data used and analyzed during the current study are available from the corresponding authors upon reasonable request.

\section{Declarations}

Competing interests

The authors declare that they have no competing interests. 


\section{References}

[1] D. Disney, T. Letavic, T. Trajkovic, T. Terashima, A. Nakagawa (2017) High-voltage integrated circuits: History, state of the art, and future prospects. IEEE Trans. Electron Devices 64(3): 659-673

[2] Nakagawa, Yasuhara, Omura, Yamaguchi, Ogura, Matsudai (1992) Prospects of high voltage power ICs on thin SOI. in IEDM Tech. Dig. 229-232

[3] T. Tian, S. Zhang, Y. F. Guo, J. Zhang, David Z. Pan, K. M. Yang (2019) A new low turn-off loss SOI lateral insulated gate bipolar transistor with buried variation of lateral doping layer. IEEE J. Electron Devices Soc. 7: 62-69

[4] E. Napoli, P. Spirito, A. G. M. Strollo, F. Frisina, L. Fragapane, D. Fagone (2002) Design of IGBT with integral freewheeling diode. IEEE Electron Device Lett. 23(9): 532-534

[5]K. Hara, S. Wada, J. Sakano, T. Oda, K. Sakurai, H. Yamashita, T. Utsumi (2014) 600V single chip inverter IC with new SOI technology. in Proc. 26th ISPSD 418-421

[6] P. A. Gough, M. R. Simpson, and V. Rumennik (1986) Fast switching lateral insulated gate transistor. in IEDM Tech. Dig. $218-221$

[7] X. Huang, D. Chang, L. Tu, and T. Q. Zheng (2018) Loss characteristics of modular multilevel converter with RC-IGBT for high voltage DC transmission. in Proc. 20th Eur. Conf. Power Electron. Appl. P.1-P.8

[8] B. Wang, L. Wang, W. Mu, M. Qin, F. Yang, J. Liu, Y. Tomoyuki, F. Tatsuhiko (2021) Thermal performances and annual damages comparison of MMC using reverse conducting IGBT and conventional IGBT module. IEEE Trans. Power Electron. 36(9): 9806-9825

[9] J. H. Chul, D. S. Byeon, J. K. Oh, M. K. Han, Y. I. Choi (2000) A fast switching SOI SA-LIGBT without NDR region. in proc. ISPSD 149-152.

[10] J. Zhu, L. Zhang, W. Sun, M. Chen, F. Zhou, M. Zhao, L. Shi, Y. Gu, S. Zhan (2016) Electrical characteristic study of an SOI-LIGBT with segmented trenches in the anode region. IEEE Trans. Electron Devices 63(5): 2003-2008

[11] L. Sun, B. Duan, and Y. Yang (2021) Novel snapback-free SOI LIGBT with shorted anode and trench barriers. IEEE Trans. Electron Devices 68(5): 2408-2413

[12] F. Udrea, U. N. K. Udugampola, K. Sheng, R. A. McMahon, G. A. J. Amaratunga, E. M. S. Narayanan, M. M. De Souza, S. Hardikar (2002) Experimental demonstration of an ultra-fast double gate inversion layer emitter transistor (DG-ILET). IEEE Electron Device Lett. 23(12): 725-727

[13] K. Sheng, U. N. K. Udugampola, G. F. W. Khoo, F. Udrea, G. A. J. Amaratunga, R. A. McMahon, E. M. S. Narayanan, M. M. De Souza, S. Hardikar (2002) Dual gate lateral inversion layer emitter transistor. in Proc. ISPSD 37-40

[14] N. K. Udugampola, R. A. McMahon, F. Udrea, and G. A. J. Amaratunga (2005) Analysis and design of the dual-gate inversion layer emitter transistor. IEEE Trans. Electron Devices 52(1): 99-105

[15] J. Wei, X. R. Luo, G. Deng, T. Sun, C. Wang, K. Zhu, W. Cui, Z. Wang, Z. Li, B. Zhang (2018) Ultrafast and low-turn-off loss lateral IEGT with a MOS-controlled shorted anode. IEEE Trans. Electron Devices 66(1): 533-538

[16] B. Yi, J. Lin, B. Zhang, J. Cheng, Y. Xiang (2020) Simulation study of a novel snapback free reverse-conducting SOI-LIGBT with embedded p-type schottky barrier diode. IEEE Trans. Electron Devices 67(5): 2058-2065

[17] S. M. Sze (2018) Physics of semiconductor devices. John Wiley \& Sons 